\title{
Overview on the model development carried out in the EURANOS project
}

\author{
W. RASKOB ${ }^{1}$
}

\begin{abstract}
The research activities under the work package CAT2 - improvement of decision support systems, focused on the one hand to improve the operability of the RODOS system but on the other hand to enhance RODOS and ARGOS with simulation models still missing in the portfolio of these two decision support systems. For RODOS, the work focused on the improvement of the atmospheric dispersion modelling in complex terrain and the improvement of the data assimilation functionalities. For both systems, the later phase models dealing with countermeasures in inhabited areas and food production systems have been considerably enhanced.
\end{abstract}

\section{Introduction}

Advanced decision support systems such as ARGOS (Hoe et al., 2002) and RODOS (Ehrhardt and Weis, 2000) contain a set of simulation models to first analyse the radiological situation and second to simulate countermeasure strategies for the early and late phase emergency actions. Finally, these results might be coupled to evaluation models that can be used to rank the most feasible countermeasures out of the many options that can be technically realised in the given situation. This conceptual cycle is presented in Figure 1 based on the RODOS system.

Basis of any simulation of the transport and dispersion of a release of radionuclides in the atmosphere are meteorological input data either monitored on site or estimated by numerical weather prognosis models for the next 48 hours. This information is digested by a local-scale Meteorological Pre-Processor MPP (Ehrhardt, 2004), the puff dispersion model RIMPUFF (Mikkelsen, 1984; Ehrhardt and Weis, 2000), implemented in RODOS and ARGOS, and the elongated puff model ATSTEP (Ehrhardt and Weis, 2000), implemented in RODOS. The applicability to farther distances in RODOS is assured with the integrated far range atmospheric dispersion model MATCH (Langner et al., 1995) with an interface for accessing meteorological data from many national and international weather services.

\footnotetext{
1 Forschungszentrum Karlsruhe GmbH, Institut für Kern- und Energietechnik, Eggenstein-Leopoldshafen, Germany.
} 


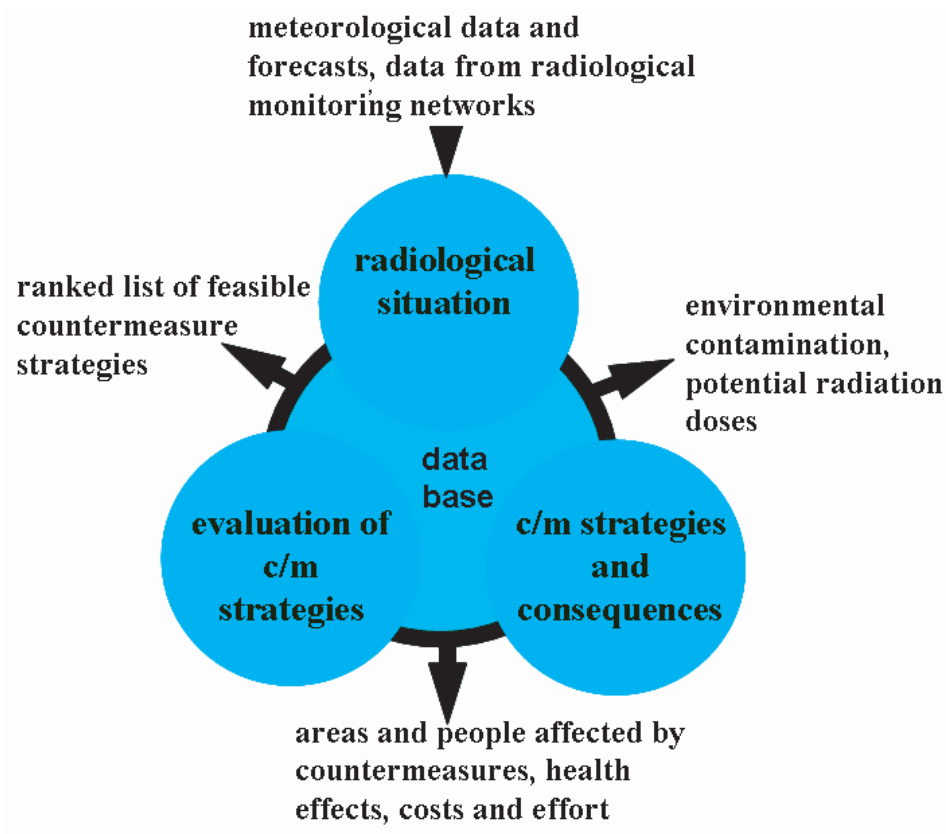

Figure 1 - Simulation cycle with first the analysis of the radiological situation, second simulation of countermeasures and finally the evaluation of the strategies.

Based on the results from the dispersion models or based on monitoring information, the exposure pathways are simulation with specific models such as the Terrestrial Food Chain and Dose Module, FDMT (Ehrhardt and Weis, 2000). This includes the simulation of the exposures from all pathways of potential importance (e.g., external exposure from a radioactive cloud, external exposure from material deposited on the ground and on the skin and clothing, and internal exposure from the inhalation of airborne material and ingestion of contaminated food).

As information from monitoring and the simulation models might differ in a real situation, approaches are necessary to deal with this information problem. To this purpose data assimilation procedures were developed and partly implemented in RODOS. Data assimilation in general is the concept of combining measured data with results of model predictions for improving the diagnostic and prognostic results. The aim is to smoothly change from pure model output to a description of the radiological situation mainly based on on-line monitoring data and measurements. Particularly, data assimilation is important for the food chain and the dose modules of RODOS, since output of these modules is the main source of information for deciding on emergency actions and long-term countermeasures. 
Once the radiological situation is analysed countermeasure strategies have to be developed. Typical areas for the simulation models are:

- sheltering;

- distribution of stable iodine tablets;

- evacuation;

- decontamination of inhabited areas;

- temporary and permanent relocation; and

- food countermeasures.

In general, simulation models are developed for the various phases separately, however, with the possibility to exchange information between the early and late phase. In the early phase, many different options for decontamination and food countermeasures are available and thus the user interaction becomes extremely important.

Whenever there is an option of two or more countermeasure strategies, a choice has to be made by the emergency management. Evaluation techniques may support this process by proposing those options and/or combinations of countermeasures, which are practicable under the actual or future conditions, and which are ranked by balancing of benefits and effort. The MAV/UT-based software package, WebHipre (Hämäläinen and Mustajoki, 1998), has been integrated in RODOS to enable users to compare and evaluate the benefits and drawbacks of different countermeasure strategies (e.g., risks, costs, feasibility, public acceptance, perceptions, social, psychological and political implications, and preferences or value concepts of decision-makers, etc.). Rules, weights, and preference functions are encoded and applied to a list of alternative countermeasures providing a ranked shortlist to decision-makers together with the rules and preferences which determined the order of the list.

\section{Status of modelling}

The objectives of the EURANOS project were to devote resources to modelling activities in all those areas, the end users requested improvements. Based on former experience in particular during the DSSNET exercises (Ehrhardt, 2005), the late phase models implemented in ARGOS and RODOS were critisised in terms of not being flexible enough to deal with complex countermeasures at different areas within one simulation session. In particular the user interaction was too complicated and limited and therefore not meeting the requirements for a comprehensive and detailed planning of measures in the later phase of an emergency. Combining this argument with the need to include at least in the intermediate phase information from monitoring and modelling, the holistic approach for the late phase models was created and realised in the working programme of EURANOS. 


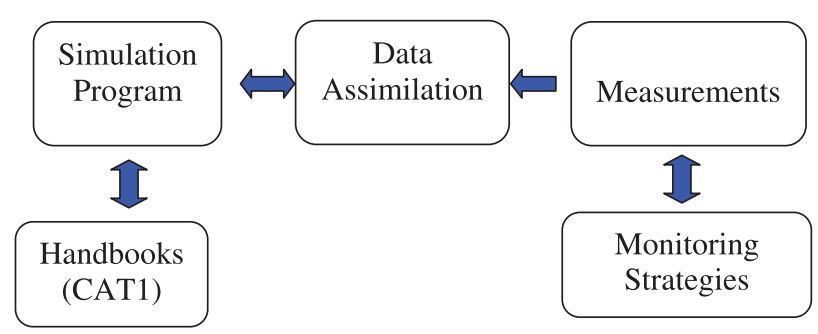

Figure 2 - Holistic approach for the modelling of countermeasures in the later phase.

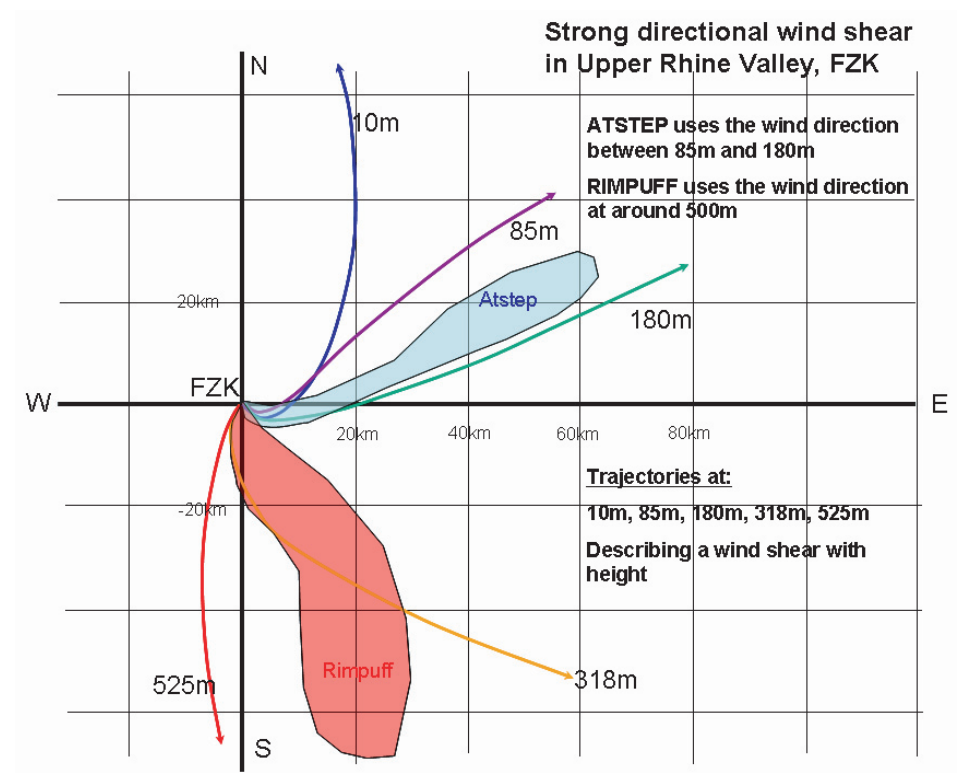

Figure 3 - Wind shear with height: different transport directions.

The simulation models developed for the later phase comprise the inhabited area model ERMIN (Charnock et al., 2009) and the food countermeasure model ArgiCP (Gering, 2010), both described in detail within this special issue.

The second major concern raised by the end users was the applicability of the atmospheric dispersion models in complex terrain. In particular when complicated meteorological conditions with wind shear in various levels, the standard Gaussian type models ATSTEP and RIMPUFF could only simulate the mean transport direction (see Fig. 3). Therefore, a particle model (DIPCOT) was requested to be implemented in RODOS. 
Besides these major areas of research work was performed to improve data assimilation and the decision aiding component.

\section{Achievements}

Details about the achievements are described in the various papers following this introduction. Therefore, this section is limited to generalised findings related to the various improvements realised. Important to note that also the hydrological model chain, that was not part of the RODOS system when starting the EURANOS project has been further developed, even if only very limited funds from the EUANOS project were devoted to this task. Details about this model chain can be found in a paper of this special issue.

\subsection{Modelling in food production and inhabited areas}

To improve the current modelling, the decision was made to implement a dynamic model that describes the behaviour of the radionuclides in the urban environment, indoors and outdoors, based on parameters and equations derived from the Chernobyl experience mainly. This new approach allowed to become flexible as the former constraint, the predefined dose libraries, were not longer used and thus the user had the full flexibility to select the countermeasure strategy and the timing of it. As result, the new European model framework - the European model for inhabited areas ERMIN was realised and integrated into both, ARGOS and RODOS, respectively.

The major improvement in simulating countermeasure options in the agricultural areas is the integration of the countermeasure simulation in the computational engine of the foodchain model AgriCP. So far, countermeasures were calculated after the simulation of the transport processes in the environment and the foodchain, but now it is part of this process. In this way, similar to ERMIN, full flexibility in defining countermeasure strategies has been achieved and the major concerns raised by the end users before the EURANOS project were successfully treated with the new models. As for ERMIN, AgriCP is implemented in ARGOS and RODOS.

\subsection{Meteorological modelling}

To implement a new particle model for the transport and dispersion of radionuclides in complex terrain, the meteorological pre-processor had to be improved that it could handle any type of dispersion model. Further, the basis for the dispersion is an accurate wind field. This was achieved with an improved diagnostic wind field model implemented into the pre-processor. The new particle model DIPCOT allows 
the simulation of atmospheric releases in the near range up to several $100 \mathrm{~km}$ and is not using much more computing time than a simple Gaussian type model due to its specific realisation of the particle characteristics. This assures that the overall performance of the RODOS system remained in the limits the end user requested (10 minutes for a prognosis over 24 hours).

The Gaussian type model RIMPUFF was expanded for applicability of up to $1000 \mathrm{~km}$. This was requested by the end user as it allows applying the near range model chain also for sites close to the home country.

Improvements for the applicability of RODOS for radiological threats was limited to an extension of the data base with relevant nuclides and the implementation of a module describing the explosion of a radiological dispersal device and thus the initial broadening of the cloud.

The long range atmospheric dispersion model MATCH was improved with data available in the numerical weather forecast systems. Further it was coupled to the RODOS source term data base.

\subsection{Data assimilation}

A data assimilation module for the inhabited areas was successfully developed. It can be used as pre-processor for the ERMIN model allowing to combine monitoring information and dispersion calculations or simply provide a better geostatistical interpolation algorithm for sparse monitoring information. Besides this improvement, the operability of data assimilation tools for the plume passage phase could not be assured. Therefore, these models - as part of the RIMPUFF model - are still in the pre-operational stage and therefore not available for operational use.

\subsection{Decision aiding components}

Work here focused on the requirements for decision making in the longer term (up to several years) and the outcome of sequential decisions depending on decisions made at each of several stages. The European handbooks for inhabited areas and food production systems were analysed and dependencies identified. This work might need to be directed back into the handbooks to provide the necessary guidance in the longer term with respect to dependencies of strategies. Additionally, approaches were derived how uncertainties, which are considered in RODOS in the various simulation modules, have to be communicated to the decision making team. 


\section{Conclusions}

Even if this summary paper only addresses some highlights of the wide range of simulation models addressed in the EURANOS project, one can clearly state that the major objective of this CAT2 activity "to improve the operability of decision support systems, in particular the RODOS system", has been achieved, taking into account the requests and comments from the end users. In particular the improvements in the later phase modelling assure now that complex countermeasure strategies can be developed without leaving the area of applicability of the simulation models.

The new dispersion model DIPCOT adds the necessary feature to be applicable in complex terrain and under complex meteorological situations within the time constraints of a real-time system which was not possible with approaches in the past.

Besides these success stories, the area of data assimilation requires still some further research to become operational. However, the successful application in the post-release phase is a big step forward towards an operational data assimilation methodology in decision support systems. This is also the case for the uncertainty handling as the realisation in a simulation model does not necessarily mean that it is accepted by the decision making team. Here again, further communication between the research and the end user community seems to be necessary.

Acknowledgment. This work has received partial financial support from the European Commission Sixth Framework Programme (Nuclear Fission/Radiation Protection) under the EURANOS integrated project: European approach to nuclear and radiological emergency management and rehabilitation strategies (Contract No: FI6R-CT-2004-508843).

\section{REFERENCES}

Charnock T.W., Jones J.A., Singer L.N., Andersson K.G., Roed J., Thykier-Nielsen S., Mikkelsen T., Astrup P., Kaiser J.C., Müller H., Pröhl G., Raskob W., Hoe S.C., Jacobsen L.H., Shou-Jensen L., Gering F. (2009) Calculating the consequences of recovery, a European Model for Inhabited Areas. Proceedings International Conference of Radioecology and Environmental Radioactivity, Bergen, Norway, 2008, Radioprotection 44, 407-412.

Ehrhardt J. (2004), Migration of RODOS to Practical Applicability, Forschungszentrum Karlsruhe, Report FZKA-7015.

Ehrhardt J. (2005) Improvement, extension and integration of operational decision support systems for nuclear emergency management (DSSNET), Report FZKA-7122.

Ehrhardt J., Weis A. (2000) RODOS: Decision Support System for Off-site Nuclear Emergency Management in Europe. European Commission, Brussels, Report EUR 19144.

Gering F., Raskob W., Charnock T. (2010) New model for agricultural countermeasures in RODOS and ARGOS, Radioprotection 45, S63-S76. 
Hämäläinen R.P., Mustajoki J. (1998) Web-HIPRE- Java Applet for Value Tree and AHP Analysis, Computer software, Systems Analysis Laboratory, Helsinki University of Technology.

Hoe S., Müller H., Gering F., Thykier-Nielsen S., Havskov Sorensen J. (2002) Argos 2001 A decision support system for nuclear emergencies, American Nuclear Society Transactions. Winter Meeting 87, 574-579.

Langner J., Persson C., Robertson L. (1995) Concentration and deposition of acidifying air pollutants over Sweden: Estimates for 1991 based on the MATCH model and observations, Water Air Soil Pollut. 85, 2021-2026.

Mikkelsen T., Larsen S.E., Thykier-Nielsen S. (1984) Description of the RISO Puff Model, Nucl. Technol. 76, 56.

\section{Web pages:}

EURANOS web site: http://www.euranos.fzk.de/

NERIS web site: http://www.eu-neris.net 